\title{
Simple quasi-analytical holonomic homogenization model for the non-linear analysis of in-plane loaded masonry panels: Part 1, meso-scale
}

\author{
G. Milani ${ }^{*}$, E. Bertolesi ${ }^{1}$ \\ 1 Department of Architecture, Built Environment and Construction Engineering ABC \\ Politecnico di Milano, Piazza Leonardo da Vinci 32, 20133 Milan, Italy \\ *corresponding author e-mail: gabriele.milani@ polimi.it
}

\begin{abstract}
A simple quasi analytical holonomic homogenization approach for the non-linear analysis of masonry walls in-plane loaded is presented. The elementary cell (REV) is discretized with 24 triangular elastic constant stress elements (bricks) and nonlinear interfaces (mortar). A holonomic behavior with softening is assumed for mortar. It is shown how the mechanical problem in the unit cell is characterized by very few displacement variables and how homogenized stress-strain behavior can be evaluated semianalytically.
\end{abstract}

Keywords: Masonry; in-plane loads; semi-analytical approach; compatible model of homogenization; holonomic non-linear model.

\section{INTRODUCTION}

Masonry is a traditional material constituted by bricks and mortar. The variability of the pattern, the shape of the blocks, and the fragile behavior of the constituent materials make the simulation of masonry a very challenging task. Macro- or micro-modeling strategies are adopted to deal with masonry over elasticity, which is usually very limited.

Macro-modeling substitutes bricks and mortar with a homogeneous, sometimes orthotropic material with softening. Abundant is the literature in this regard, see e.g. [1]. Macro-modelling allows studying even large scale structures without the need of meshing separately bricks and mortar. Nevertheless, the calibration of model parameters is typically done through expensive experimental campaigns.

The alternative is micro-modeling, which is characterized by a separate discretization of mortar and bricks. The reduction of joints to interfaces [2] helps in limiting variables, but the approach still remains demanding.

In such a context, homogenization [3]-[6] is a fair compromise between micro- and macro-modelling, because it allows in principle to perform structural non-linear analyses without a distinct representation of bricks and mortar, still considering their mechanical properties and texture at a cell level. Homogenization is essentially an averaging procedure performed at a meso-scale on a representative element of volume (REV), which generates masonry by repetition. On the REV, a Boundary Value Problem BVP is formulated, allowing an estimation of the expected average masonry behavior to be used at structural level.

In this paper, a simplified homogenization two-step model is proposed for the non-linear structural analysis of masonry walls in-plane loaded. The first step is applied at the meso-scale, where the assemblage of bricks and mortar in the REV is substituted with a macroscopic equivalent material through a so called compatible identification. The unit cell is meshed by means of 24 triangular elastic plane stress elements (bricks) and interfaces (mortar). For interfaces, a piecewise linear and an exponential law formally identical to an improved version of the Xu-Needleman law are implemented. Such relationships are characterized by post-peak softening, eventually with a coupling between normal and shear stresses in the case of Xu-Needleman. The second step, reported in detail in Part 2, is performed at a structural level, and relies into the implementation of the homogenized stress-strain relationships into either a FE code dealing with softening materials (nested multi-scale technique) or a rigid element approach (RBSM) where contiguous rigid elements are connected by shear and normal non-linear homogenized springs.

\section{THE NUMERICAL MODEL}

The basic features of the REV model are depicted in FIGURE 1. The REV middle plane is discretized with 24 plane stress elastic triangular elements (bricks) and holonomic softening interfaces (mortar with zero thickness). Under the application of a single stretching along horizontal direction $\left(E_{x x} \neq 0\right.$ in the homogenized strain tensor), only $1 / 4$ of the unit cell can be considered, with the behavior of elements 4,5 and 6 equal to those of elements 3,2 and 1 respectively. Under such assumptions, it has been shown in [7], that after writing properly both equilibrium and compatibility equations and after proper manipulation of such relationships, the following equations are obtained to solve the mechanical problem on the REV: 
(a) Curve I $\eta=g(\xi): \eta=\frac{2 H}{v_{b} L}\left[U_{x}^{0}-\xi-L \frac{1-v_{b}^{2}}{E_{b}} f_{n}^{I}(\xi)-\frac{L^{2}}{2 H} \frac{1-v_{b}^{2}}{E_{b}} f_{t}^{I I}(\xi)\right]$

(b) Curve II $\xi=f(\eta): \xi=U_{x}^{0}+\frac{L}{2 v_{b} H}\left[-\eta-2 \frac{1-v_{b}^{2}}{E_{b}} H f_{n}^{I I}(\eta)\right]$

In Eq. (1), $U_{x}^{0}$ indicate an imposed boundary horizontal displacement, $\xi=U_{x}^{0}-U_{x}^{9}, \eta=U_{y}^{5}+U_{y}^{6}, U_{x}^{i}\left(U_{y}^{i}\right)$ is the i-th node unknown horizontal (vertical) displacement, $f_{n}^{I, I I}\left(f_{t}^{I, I I}\right)$ is the joint (I: head, II: bed) normal (shear) stress, $E_{b}\left(v_{b}\right)$ is the brick Young modulus (Poisson's ratio).

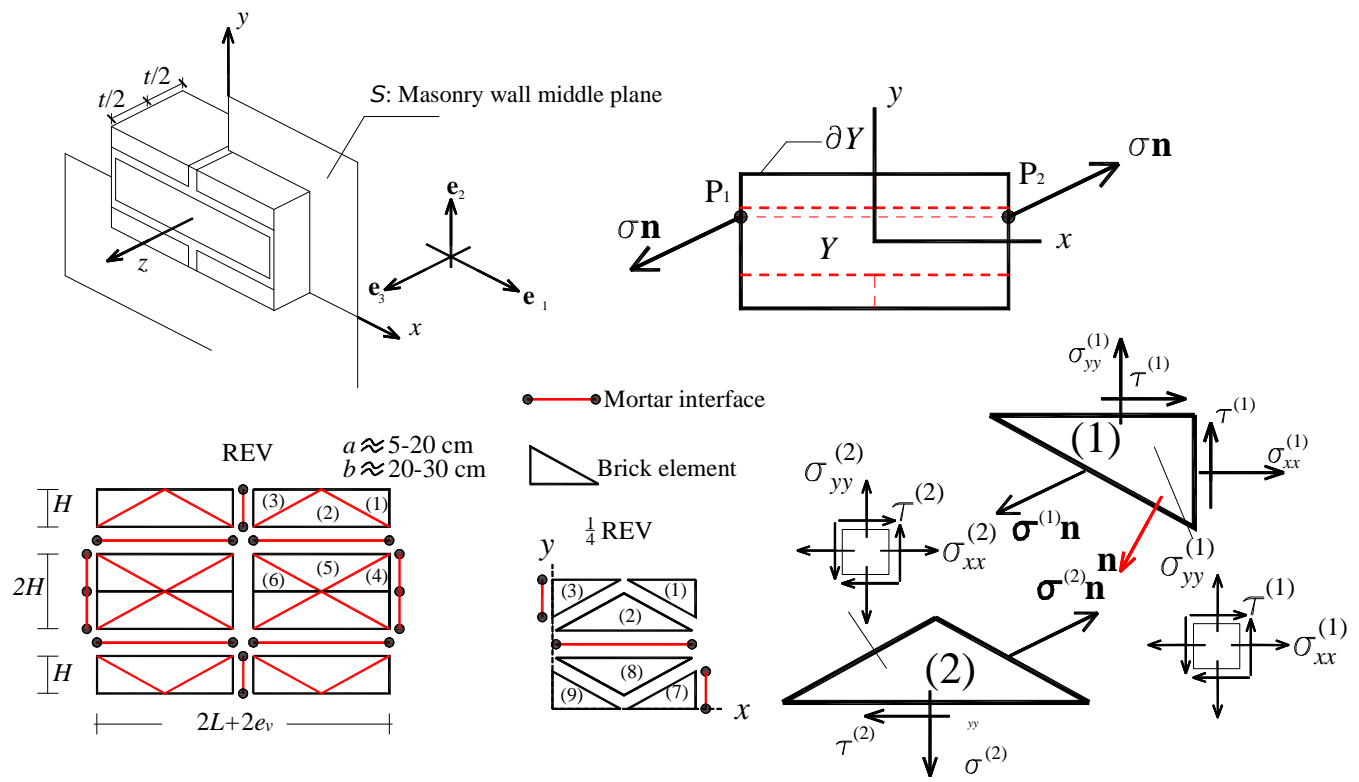

FIGURE 1: The compatible homogenization presented. REV mesh with 24 CST brick elastic elements and holonomic mortar interfaces. Anti-periodicity of the micro-stress field.

Eq. ( 1 ) is a system of non-linear equations that can be solved graphically as follows, see FIGURE 2:

1) Assign a value for $\xi$ in equation ( 1 )(a) and find immediately the corresponding value of $\eta$. Curve ( 1 )(a) can thus be plotted in the $\xi-\eta$ plane selecting a suitable range for $\xi$. Since $\xi$ is the tangential jump of displacements of the horizontal joint, typically the range to inspect is $\xi \in\left[\begin{array}{ll}0 & \Delta_{t}^{u l}\end{array}\right]$, where $\Delta_{t}^{u l}$ is the ultimate tangential jump of displacement of the interface.

2) Assign a value for $\boldsymbol{\eta}$ in equation ( 1 )(b) and find immediately the corresponding value of $\xi$. Similarly to Curve I,

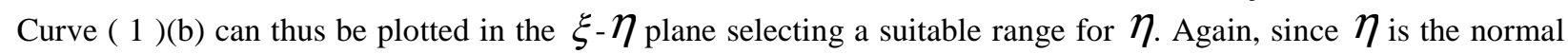
jump of displacements of the horizontal joint, the range to inspect is $\eta \in\left[\begin{array}{ll}0 & \Delta_{n}^{u l}\end{array}\right]$, where $\Delta_{n}^{u l}$ is the ultimate normal jump of displacement of the interface.

3) The intersection between Curve I and Curve II allows the graphical determination of $\xi$ - $\eta_{\text {values. }}$

When shear and normal behaviors of the interfaces are coupled, analogous relations are derived:

(a) $\eta=\frac{2 H}{v_{b} L}\left[U_{x}^{0}-\xi-L \frac{1-v_{b}^{2}}{E_{b}} f_{n}^{I}(\xi)-\frac{L^{2}}{2 H} \frac{1-v_{b}^{2}}{E_{b}} f_{t}^{I I}(\xi, \eta)\right]$

(b) $\xi=U_{x}^{0}+\frac{L}{2 v_{b} H}\left[-\eta-2 \frac{1-v_{b}^{2}}{E_{b}} H f_{n}^{I I}(\xi, \eta)\right]$

In this latter case, however, the graphical procedure to determine the solution point is slightly more cumbersome and requires a recursive approach as follows:

4) Assign a value for $\xi$ in equation ( 2 (a) with $\eta_{=0}$ in $f_{t}^{I I}(\xi, \eta)$ and find an updated value for $\eta$, say $\eta^{i}$. Put $\eta^{i}$ into $f_{t}^{I I}(\xi, \eta)$ and, through ( 2 )(a), estimate again $\eta_{=} \eta^{i+1}$. Repeat until $\eta^{i} \approx \eta^{i+1}$. Curve ( 1 )(a) is thus plotted

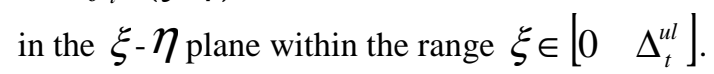




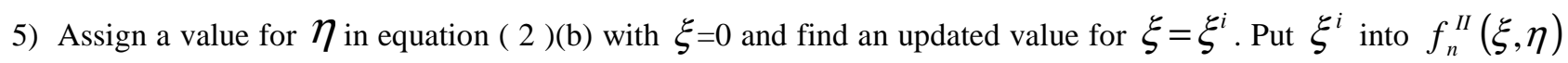

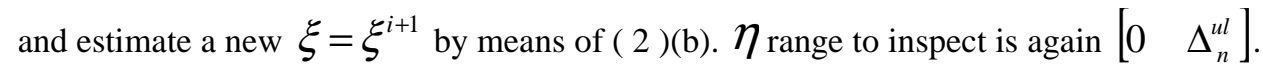

6) $\xi-\eta$ values are estimated at the intersection between Curve I and Curve II.
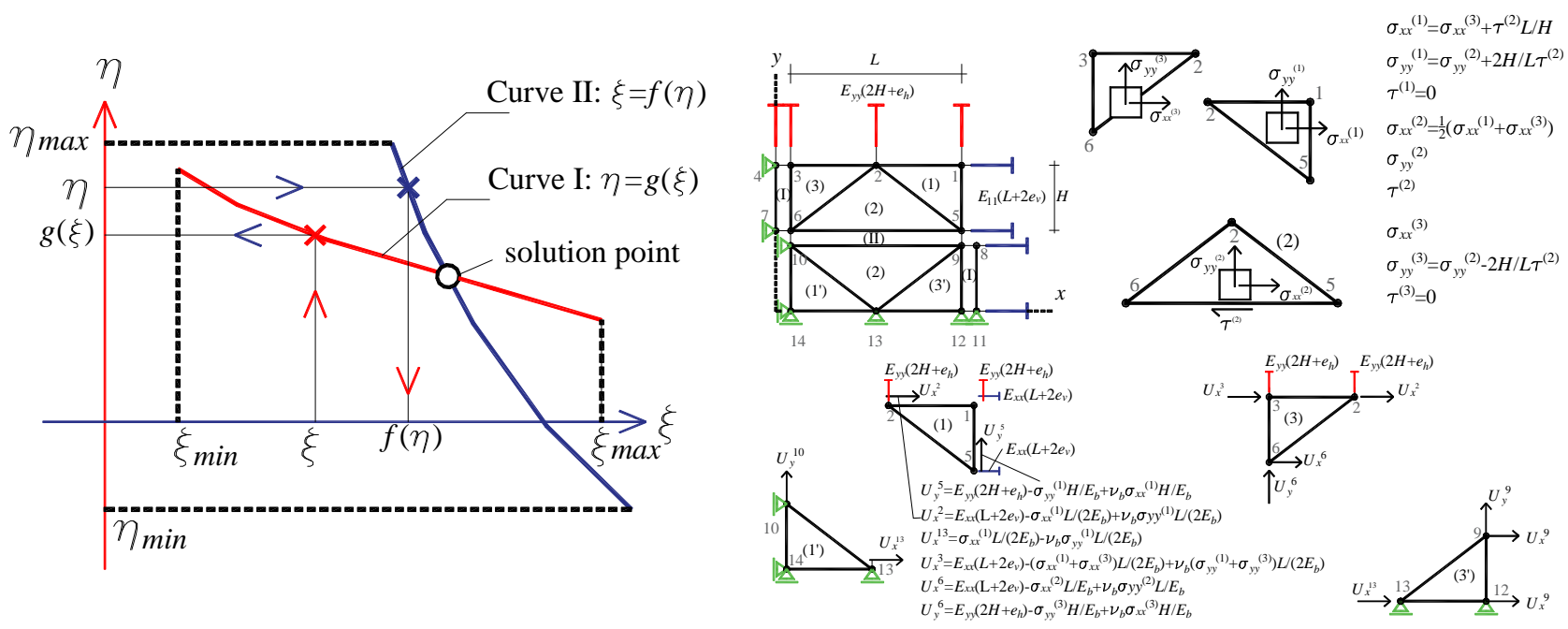

FIGURE 2: Graphical solution in the biaxial strain state (left) and biaxial strain state problem (right).

When a biaxial strain state is applied to the unit cell, i.e. with both $E_{x x} \neq 0$ and $E_{y y} \neq 0$, it can be shown that equations ( 1 ) slightly modifies into:

$$
\begin{aligned}
& \text { Curve I } \eta=g(\xi): \eta=U_{y}^{0}+\frac{2 H}{v_{b} L}\left[U_{x}^{0}-\xi-L \frac{1-v_{b}^{2}}{E_{b}} f_{n}^{I}(\xi)-\frac{L^{2}}{2 H} \frac{1-v_{b}^{2}}{E_{b}} f_{t}^{I I}(\xi)\right] \\
& \text { Curve II } \xi=f(\eta): \xi=U_{x}^{0}+\frac{L}{2 v_{b} H}\left[U_{o}^{y}-\eta-2 \frac{1-v_{b}^{2}}{E_{b}} H f_{n}^{I I}(\eta)\right]
\end{aligned}
$$

where $U_{y}^{0}$ is an applied vertical boundary displacement, representing $E_{y y} \neq 0$, according to the compatible identification procedure adopted. The solution strategy for the non-linear system of equations ( 3 ) is identical to that adopted for problem ( 2 ). For shear, with the positions $U_{x}^{5}+U_{x}^{6}=\xi^{t}, U_{y}^{3}=\eta^{t}, E_{x y}\left(2 H+e_{h}\right)=\bar{U}_{x}^{t}$, $E_{y x}\left(L+2 e_{v}\right)=\bar{U}_{y}^{t}, \frac{\tau^{(1)} e_{v}}{G_{b}}=\kappa$, the following equations hold (it is interesting to notice that ( 4 ) is a system of nonlinear equations into the three variables $\xi^{t}, \eta^{t}$ and $\kappa$, again solved with a simple iterative strategy fully explained in [7]:

$$
\begin{aligned}
& \xi^{t}=2 \bar{U}_{x}^{t}-\frac{H}{G_{b}}\left[-\frac{\bar{U}_{y}^{t}-\eta^{t}}{L / 2} G_{b}+2 f_{t}^{I}\left(\eta^{t}\right)-\frac{4 H}{L} f_{n}^{I I}\left(\eta^{t}-\kappa\right)+2 \frac{\kappa}{L} G_{b}\right] \\
& \eta^{t}=\bar{U}_{y}^{t}-\frac{L}{2 G_{b}}\left[2 f_{t}^{I I}\left(\xi^{t}-\bar{U}_{x}^{t}\right)-\frac{2 \bar{U}_{x}^{t}-\xi^{t}}{H} G_{b}+2 \frac{\kappa}{L} G_{b}\right] \\
& \kappa=\frac{e_{v}}{G_{b}}\left[2 f_{t}^{I I}\left(\xi^{t}-\bar{U}_{x}^{t}\right)-f_{t}^{I}\left(\eta^{t}\right)\right]
\end{aligned}
$$

\section{RESULTS}

Some concise results are represented in FIGURE 3 for a running bond masonry with mechanical properties as in TABLE I. A comprehensive discussion is again provided in [7] and is not reported here for the sake of conciseness. In FIGURE 3 the homogenized stress-strain relationships obtained with the model are represented in case of horizontal (top-left sub-figure) and vertical (top-right sub-figure) stretching. Both the response obtained adopting a multi-linear and a Xu-Needleman interface behavior is represented. As can be noted, both interface models provide similar homogenized results, with an expected extra-resistance of the REV for horizontal stretching (contribution of the bed joint in shear). Multi-linear and Xu-Needleman model provide almost superimposable homogenized curves and in 
uniaxial stretching it is also interesting to notice the presence of a biaxial stress behavior, a consequence of the elastic Poisson's effect on bricks, which intuitively tends to vanish when damage on joints proceeds. The same figure (bottom sub-figures) shows the iterative solution found plotting Eqs. ( 3 ), assuming for joints a Xu-Needleman law. As can be observed, after only 3 iterations, the solution is already found with negligible error.

TABLE I: Mechanical properties assumed for the constituent materials( for mortar joints eh=10 $\mathrm{mm}$ ) in the benchmark discussed.

\begin{tabular}{|c|c|c|c|c|c|c|c|c|c|}
\hline \multicolumn{9}{|c|}{ Multi-linear model } & \multicolumn{4}{c|}{ Xu-Needleman model } \\
\hline$E_{m}$ & $G_{m}$ & $\mathrm{ft}$ & $\mathrm{c}$ & $\Delta_{n u}$ & $\Delta_{t u}$ & $\phi_{n}$ & $\boldsymbol{\delta}_{n}$ & $\phi_{t}$ & $\boldsymbol{\delta}_{t}$ \\
\hline$[\mathrm{MPa}]$ & {$[\mathrm{MPa}]$} & {$[\mathrm{MPa}]$} & {$[\mathrm{MPa}]$} & {$[\mathrm{mm}]$} & {$[\mathrm{mm}]$} & {$[\mathrm{N} / \mathrm{mm}]$} & {$[\mathrm{mm}]$} & {$[\mathrm{N} / \mathrm{mm}]$} & {$[\mathrm{mm}]$} \\
\hline 800 & $0.4 E_{m}$ & 0.25 & $1.35 \mathrm{ft}$ & $6 \mathrm{eh} \mathrm{ft} / E_{m}$ & $3 \mathrm{eh} \mathrm{c} / G_{m}$ & 0.0028 & 0.0042 & 0.0060 & 0.0160 \\
\hline
\end{tabular}

Bricks linear elastic ( $E_{b}=16700 \mathrm{MPa}, v_{b}=0.15$ ), ft: mortar tensile cutoff, c: mortar cohesion, $\Delta_{n u}$ ultimate displacement of joints for normal stresses, $\Delta_{t u}$ ultimate displacement of joints for shear stresses.
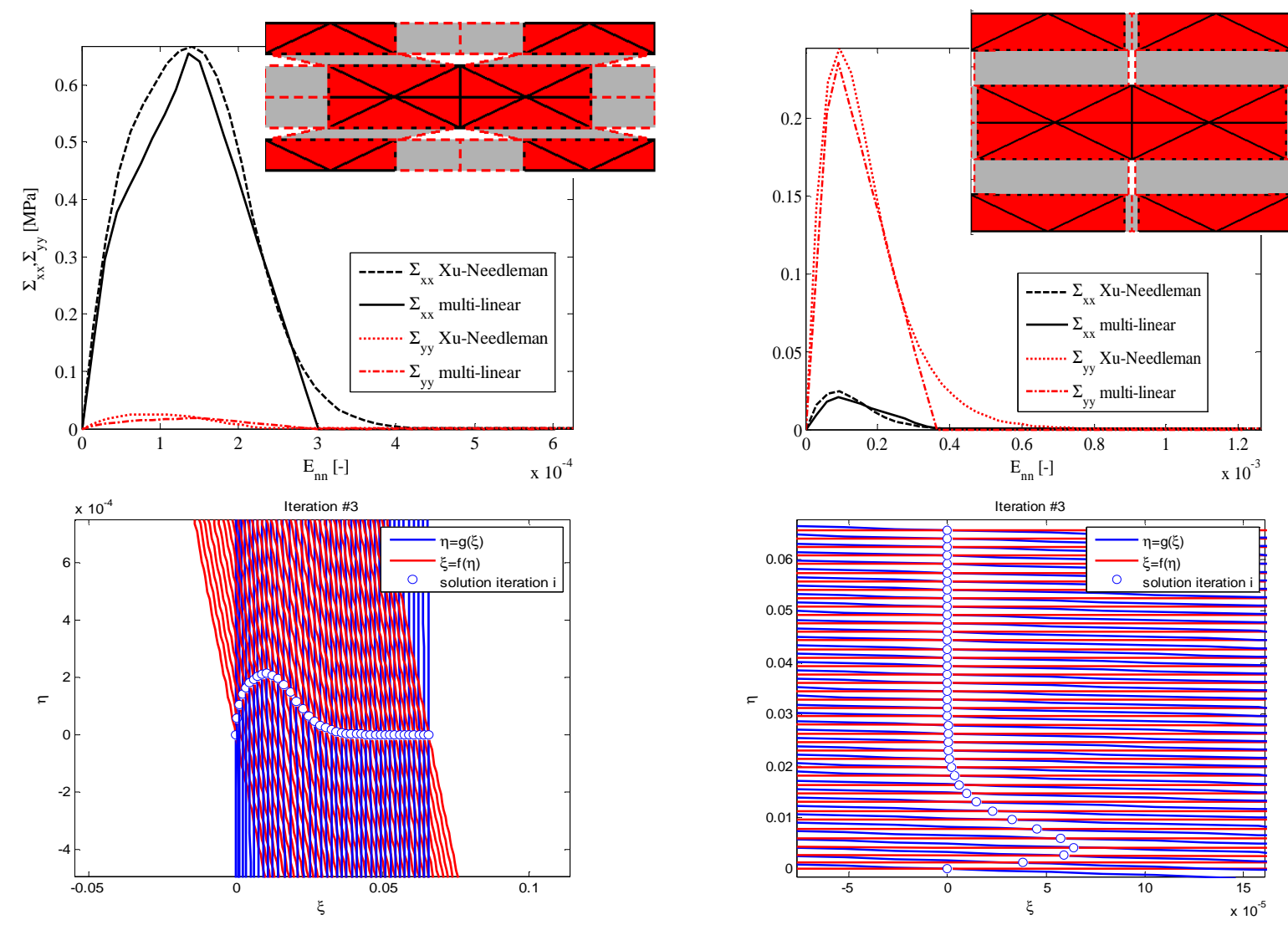

FIGURE 2: Graphical solution in the biaxial strain state (left) and biaxial strain state problem (right).

\section{REFERENCES}

[1] Berto L, Saetta A, Scotta R, Vitaliani R. An orthotropic damage model for masonry structures. Int J Numer Methods Engng 2002; 55: 127-57.

[2] Sutcliffe DJ, Yu HS, Page AW. Lower bound limit analysis of unreinforced masonry shear walls. Computers \& Structures 2001; 79: 1295-1312.

[3] Milani G. Four approaches to determine masonry strength domain. Proceedings of ICE, Engineering and Computational Mechanics 2015; 168(3): 99-121.

[4] Milani G. Simple homogenization model for the non-linear analysis of in-plane loaded masonry walls. Computers \& Structures 2011; 89: 1586-1601.

[5] Milani G, Lourenço PB, Tralli A. Homogenised limit analysis of masonry walls. Part I: failure surfaces. Computers \& Structures 2006; 84(3-4): 166-180.

[6] Milani G, Lourenço PB. Monte Carlo homogenized limit analysis model for randomly assembled blocks in-plane loaded. Computational Mechanics 2010; 46(6): 827-849.

[7] Milani G, Bertolesi E. Holonomic homogenized approach for the non-linear analysis of in-plane loaded masonry panels. Under review 2016. 